\title{
INNOVATIVE DESIGN OF «COBOT» TYPE SMART MULTIAPLICATIVE PLATFORMS USED IN THE INDUSTRY 4.0 AND MADE BY INCDMTM - BUCHAREST
}

\author{
Gheorghe Gheorghe \\ ${ }^{1}$ National Institute of Research and Development for Mechatronics and Measurement \\ Technique - INCDMTM, Bucharest, Romania \\ Email: geocefin@yahoo.com
}

\begin{abstract}
The scientific paper " Innovative Design of «COBOT» Type Smart Multiaplicative Platforms used in the Industry 4.0 and Made by INCDMTM - Bucharest" with micro hexapod ( $\mathrm{x}, \mathrm{y}, \mathrm{z}$, $\theta \mathrm{X}, \theta \mathrm{Y}, \theta \mathrm{Z}$ ) and ultraprecise probe for measuring parts and 3D laser scanning 3D, which are used in the smart processes of process of measuring in smart industrial processes.
\end{abstract}

Keywords: Smart Platform; Robot \& Microsystem Hexapod; Ultra-Precise Probe; 3D Laser Scanning; Smart Industry.

\section{Introduction}

Based on the development of new knowledge, new innovative design solutions and modern adaptive concepts there were conceived technical + technological parts of smart measurement technology platforms and smart control platforms integrated in the Smart industry (4.0).

In all the constructions presented in the paper, collaborative smart processes with other robots and other operators have been integrated to support the Smart Industry (4.0).

\section{2."COBOT" Type Multiaplicative Smart Platform with Robot \& Hexapod Microsystem and Ultraprecise Probe for Measuring Parts with a Mass of $3 \mathrm{Kg}$}

In the application depicted in the figure below, a collaborative robotic platform (1) for the smart measurement of functional objects with a mass of 3 $\mathrm{Kg}$ is presented, which integrates two robotic systems, in a serial-parallel display (Patent ID: $A$ 00610), providing 11 degrees of freedom (11DOF) (6DOF universal robot (1.6), 6DOF hexapod robot (1.12) of which $1 D O F$ is common with that of the universal robot - respectively the final effector) and allows the positioning in space, in two stages, of the final effector, respectively an ultra-precise transducer probe (1.13), by orienting it throughout the general workspace, with the positioning accuracy of the universal robot $(0.2 \mathrm{~mm})$ and the local orientation with nanometric precision $(7 \mathrm{~nm})$, of the final effector probe with the hexapod robotic system $( \pm 7 \mathrm{~mm})$, in the vicinity of the point where the measurement is intended.

By using a parallel structure specific to the (hexapod) Stewart platform we ensure a much more accurate smart positioning (about 28x10 3 times more accurate) than that of the serial structures that position the palpation transducer and the effector of a single robot.

The smart multi-application platform type "Cobot" realizes the remote control of mechatronic cyber-mix platforms type "multi-application Cobot" (1) by bidirectional data transmission and realization of remote control, telemonitoring and teleservice functions through the cyberspace (2) in the Centre Remote control and Teleservice (3), using specialized programming and visualization software.

The assembly is equipped with a multisensory system consisting of: smart colour sensor (1.3), smart video identification system components (1.4), IP camera (1.5), smart camera COMPACT VISION (1.7). The fusion of data from the multisensory system together with those from the final effector used depending on the application, allows the performance of a variety of smart tasks, with a high degree of adaptability and flexibility. 
Innovative Design of «COBOT» Type Smart Multiaplicative Platforms used in the Industry 4.0 and Made by INCDMTM - Bucharest

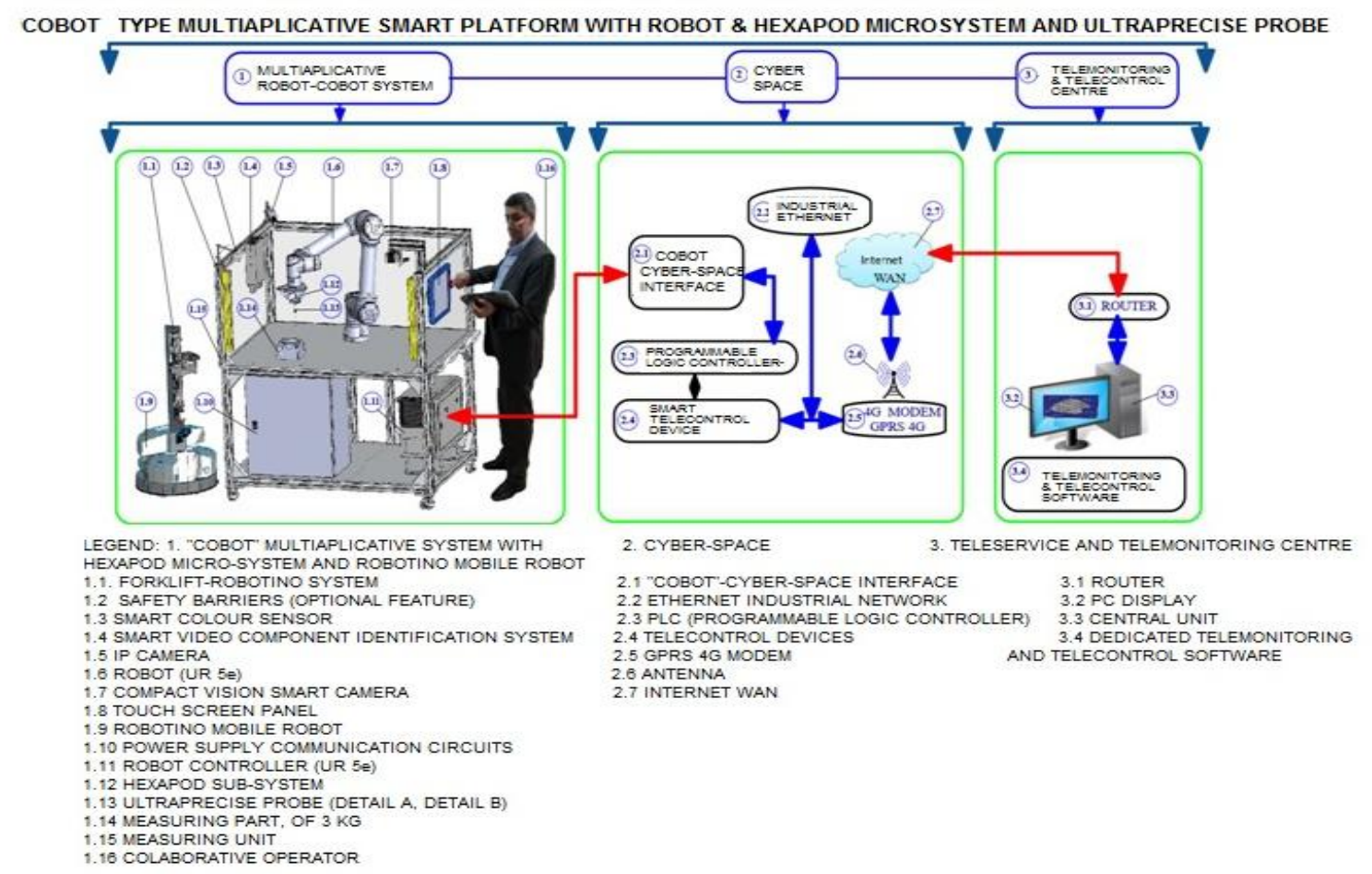

Figure 1
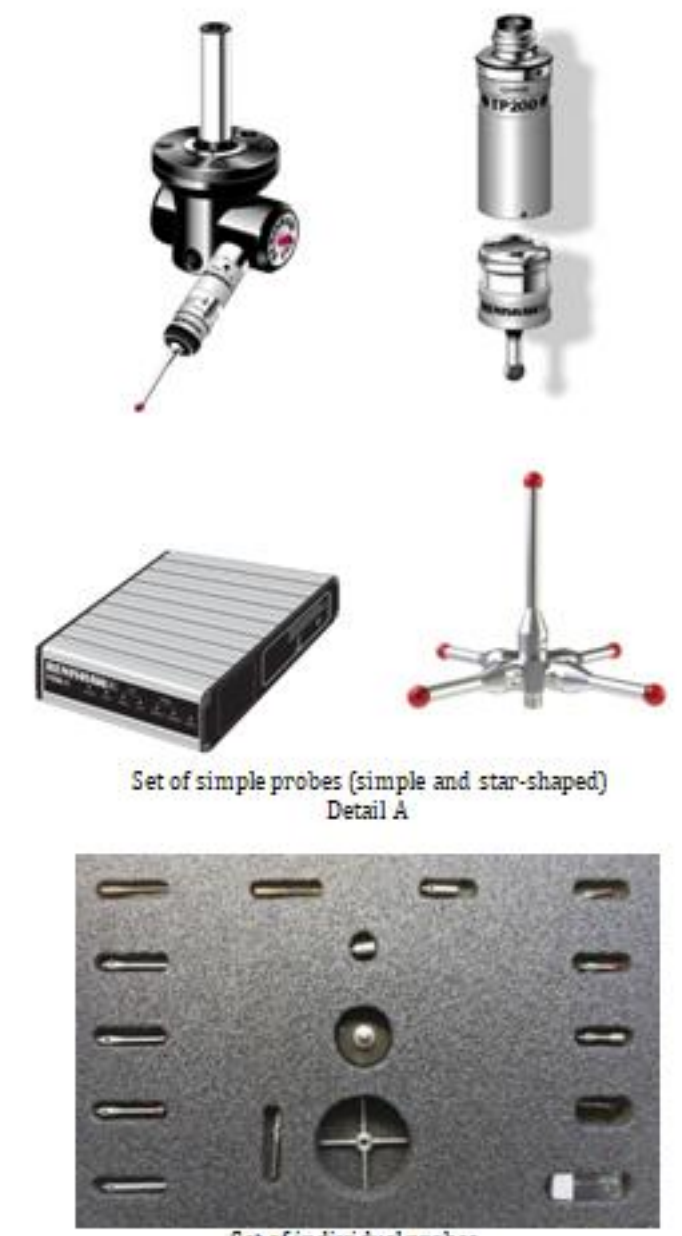

Set of individual probes

Detail B

Figure 2 
A mobile smart robotic platform (Robotino) (1.9), equipped with WiFi communication system, performs the collaborative function of supplying parts \subassemblies subjected to the adaptive process of smart analysis \measurement, by fixing them on the rigid mass (1.14) using a mechatronic system Forklift-Robot (1.1). The synchronization with the cobotic platform as well as the communication with the remote-control centre is done via WiFi, directly in the Wan Internet network.

The system is additionally protected by infrared safety barriers (1.2) and communicates with the special equipment in the cabinet (1.11) both with the internal industrial bus (2.2) and with the WAN Internet network via the 4G GPRS modem. This communication connection connects to the remotecontrol centre (3) provided with a PC central unit (3.3) connected to the router (3.1) and on which the specialized software (3.4) runs.

The system can also be operated locally, using a pre-installed program by emulating working positions using the Touch-Screen Panel (1.8). The positioning of the final effector (3D scanner) can be done in a well-defined area of the entire workspace of the robot with 6 degrees of freedom (6DOF).

During the measurement process, the position information values - the measured values - are transmitted in numerical vector data packages for complex processing both in real time and subsequent processing too. In addition to the innovative performance of robotic platforms, operator safety is another necessary prerequisite of successful operations under smart manufacturing process.

To avoid risky situations, before the emergence of robots, industrial robots have been kept away from workers through a series of protection measures since the beginning of the history of automation. Collaborative robots have completely changed the paradigm and allowed the implementation of cobotic platforms, without a safety infrastructure that requires high costs and space.

The main competitive advantages of UR cobots include easy operation, fast deployment and an interface that allows the collaborative operator (1.16) to take on a form of intuitive programming, which can also be performed by direct physical contact with the robotic arm or via the touch-screen. Cobots are able to work in close proximity to humans without safety barriers, but this approach requires full control over the speed, force, power, and momentum generated by the cobots during their operations. Even though UR cobots are at the top of safety standards, the multi-application cobot platform has also been provided with optical safety barriers, as an additional adaptive function for a wide range of applications.

The connection to the cyberspace (2) is made through a specialized interface (2.1) that allows communication with the robot controller (1.11) the data being adapted to be transferred to a Programmable Logic (PLC) (2.3) used to automate mechatronic processes and for storing the working variables of the specific control program, as well as to receive - in a synchronized way - information from the smart remote control equipment (2.4) implemented either with microcontroller via RISC architecture, or via FPGA, and which connect the PLC and the 4G telecommunication modem using a specific RS232 or USB protocol.

Various public or private networks can be used to access the 4G connection. Various modems (2.3) can be used for connectivity, and they are all compatible with VPN protocols. Event-driven, or cyclic data processing is performed using special remotecontrol protocols and allows the operating personnel to efficiently manage the process as a whole, or in detail. The Remote-Control Centre (3) provides the connection to the WAN Internet (2.7) using a router (3.1) equipped with VPN function to ensure a secure data encryption.

A second level of security can be studied by using its own encryption algorithm and by specifically configuring the FIREWALL function of the operating system on the PC (OS: Windows or Linux). The computer (3.3) in the Telemonitoring Centre (3) runs a software specially designed to perform the functions of remote control, telemonitoring and teleservice.

\section{Robot System - Multiaplicative cOBOT (with 3D Laser Scanner)}

In the application in the figure below, the Universal Collaborative Robot (1.6) performs a positioning of a 3D smart laser scanner (1.12), controlled by a PC with specific software located in the telemonitoring and remote-control centre (3) and used to perform the function of smart measurement / control of parts (1.13).

The smart multi-application platform type "Cobot" realizes the remote control of mechatronic cyber-mix platforms type "multi-application Cobot" (1) by bidirectional data transmission and realization of remote control, telemonitoring and teleservice functions through the cyberspace (2) in the Centre Remote control and Teleservice (3), using specialized programming and visualization software. 


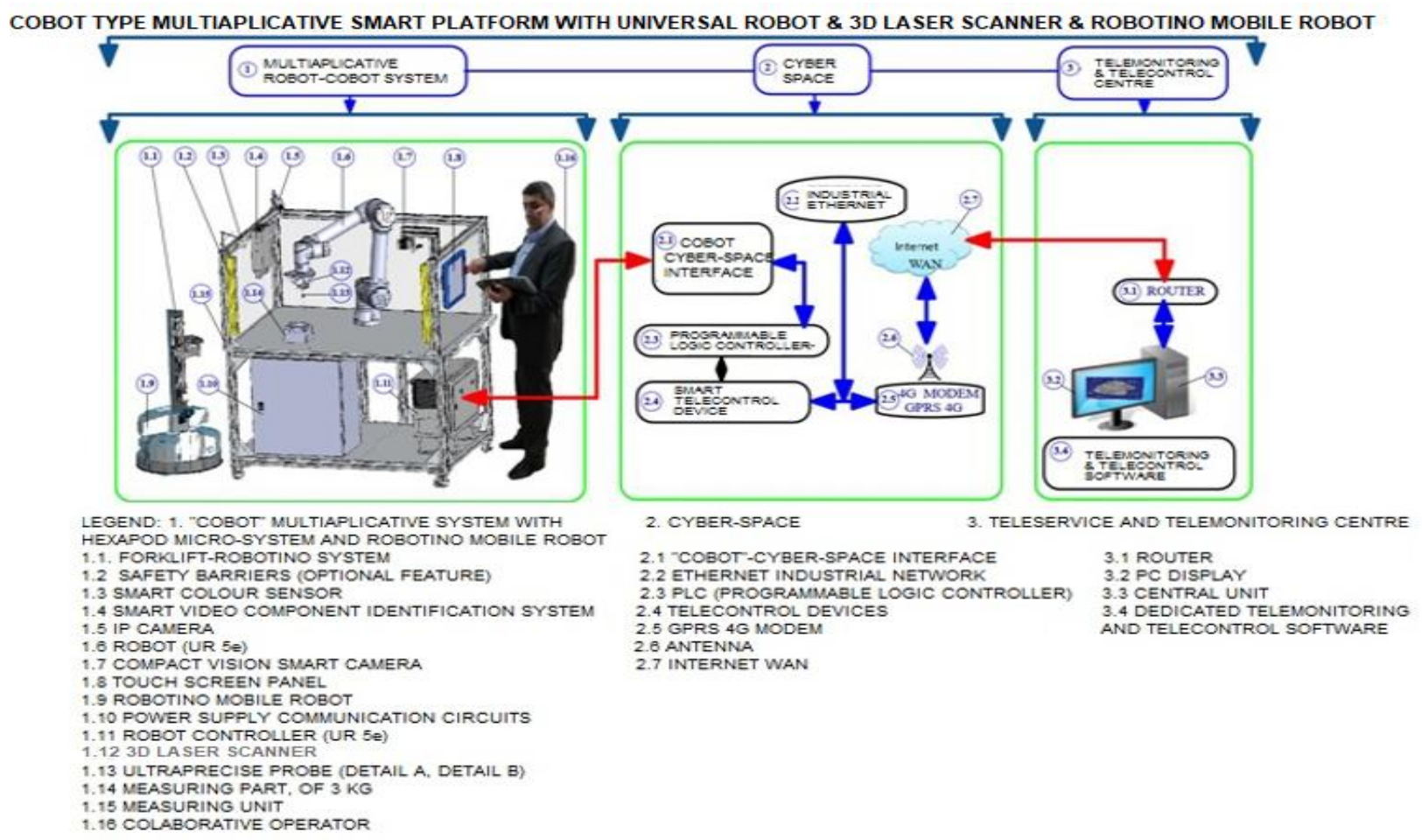

Figure 3

The assembly is equipped with a multisensory system consisting of: a smart colour sensor (1.3), a smart video component identification system (1.4), an IP camera (1.5), a «COMPACT VISION» smart camera (1.7). The fusion of data from the multisensory system together with those from the final effector used depending on the application, allows the performance of a variety of smart tasks, along with a high degree of adaptability and flexibility.

A mobile smart robotic platform (Robotino) (1.9), equipped with WiFi communication system, performs the collaborative function of supplying parts/ subassemblies subjected to the adaptive process of smart analysis / measurement, by fixing them on the rigid mass (1.14) using a Forklift-Robot mechatronic system (1.1). The synchronization with the cobotic platform, as well as the communication with the remote-control centre is done via $\mathrm{WiFi}$, directly in the Wan Internet network.

The system is additionally protected by infrared safety barriers (1.2) and communicates with the special equipment in the cabinet (1.11) both with the internal industrial bus (2.2) and with the WAN Internet network via the 4G GPRS modem.
This communication connection connects to the remote-control centre (3) provided with a PC central unit (3.3) connected to the router (3.1) and on which the specialized software (3.4) runs.

The system can also be operated locally, using a pre-installed program by emulating working positions using the Touch-Screen Panel (1.8). The positioning of the final effector (3D scanner) can be done in a well-defined area of the entire workspace of the robot with 6 degrees of freedom (6DOF).

During the measurement process, the position information values - the measured values - are transmitted in numerical vector data packages for complex processing both in real time and subsequent processing too.

In addition to the innovative performance of robotic platforms, operator safety is another necessary prerequisite of successful operations under smart manufacturing process. To avoid risky situations, before the emergence of robots, industrial robots have been kept away from workers through a series of protection measures since the beginning of the history of automation. Collaborative robots have completely changed the paradigm and allowed the implementation of cobotic platforms, without a safety infrastructure that requires high costs and space. 
The main competitive advantages of UR cobots include easy operation, fast deployment and an interface that allows the collaborative operator (1.16) to take on a form of intuitive programming, which can also be performed by direct physical contact with the robotic arm or via the touch-screen. Cobots are able to work in close proximity to humans without safety barriers, but this approach requires full control over the speed, force, power, and momentum generated by the cobots during their operations. Even though UR cobots are at the top of safety standards, the multi-application cobot platform has also been provided with optical safety barriers, as an additional adaptive function for a wide range of applications.

The connection to the cyberspace (2) is made through a specialized interface (2.1) that allows communication with the robot controller (1.11) the data being adapted to be transferred to a Programmable Logic (PLC) (2.3) used to automate mechatronic processes and for storing the working variables of the specific control program, as well as to receive - in a synchronized way - information from the smart remote control equipment (2.4) implemented either with microcontroller via RISC architecture, or via FPGA, and which connect the PLC and the $4 \mathrm{G}$ telecommunication modem using a specific RS232 or USB protocol.

Various public or private networks can be used to access the 4G connection. Various modems (2.3) can be used for connectivity, and they are all compatible with VPN protocols. Event-driven, or cyclic data processing is performed using special remotecontrol protocols and allows the operating personnel to efficiently manage the process as a whole, or in detail.

The Remote-Control Centre (3) provides the connection to the WAN Internet (2.7) using a router (3.1) equipped with VPN function to ensure a secure data encryption.

A second level of security can be studied by using its own encryption algorithm and by specifically configuring the FIREWALL function of the operating system on the PC (OS: Windows or Linux). The computer (3.3) in the Telemonitoring Centre (3) runs a software specially designed to perform the functions of remote control, telemonitoring and teleservice.

\section{4. "COBOT" Type Multiaplicative Smart Platform with Robot \& Hexapod Microsystem and Ultraprecise Probe for Measuring Parts with a Mass of $5 \mathrm{Kg}$}

In the application depicted in the figure below, a collaborative robotic platform (1) for the smart measurement of functional objects with a mass of 5 $\mathrm{Kg}$ is presented, which integrates two robotic systems, in a serial-parallel display (Patent ID: $A$ 00610), providing 11 degrees of freedom (11DOF) (6DOF universal robot (1.6), 6DOF hexapod robot (1.12) of which 1DOF is common with that of the universal robot - respectively the final effector) and allows the positioning in space, in two stages, of the final effector, respectively an ultra-precise transducer probe (1.13), by orienting it throughout the general workspace, with the positioning accuracy of the universal robot $(0.2 \mathrm{~mm})$ and the local orientation with nanometric precision $(7 \mathrm{~nm})$, of the final effector probe with the hexapod robotic system $( \pm 7 \mathrm{~mm})$, in the vicinity of the point where the measurement is intended.

The smart multi-application platform type "Cobot" realizes the remote control of mechatronic cyber-mix platforms type "multi-application Cobot" (1) by bidirectional data transmission and realization of remote control, telemonitoring and teleservice functions through the cyberspace (2) in the Centre Remote control and Teleservice (3), using specialized programming and visualization software.

The assembly is equipped with a multisensory system consisting of: a smart colour sensor (1.3), a smart video component identification system (1.4), an IP camera (1.5), a «COMPACT VISION» smart camera (1.7). The fusion of data from the multisensory system together with those from the final ffector used depending on the application, allows the performance of a variety of smart tasks, along with a high degree of adaptability and flexibility.

A mobile smart robotic platform (Robotino) (1.9), equipped with WiFi communication system, performs the collaborative function of supplying parts/ subassemblies subjected to the adaptive process of smart analysis / measurement, by fixing them on the rigid mass (1.14) using a Forklift-Robot mechatronic system (1.1).

The synchronization with the cobotic platform, as well as the communication with the remote-control centre is done via $\mathrm{WiFi}$, directly in the Wan Internet network. 


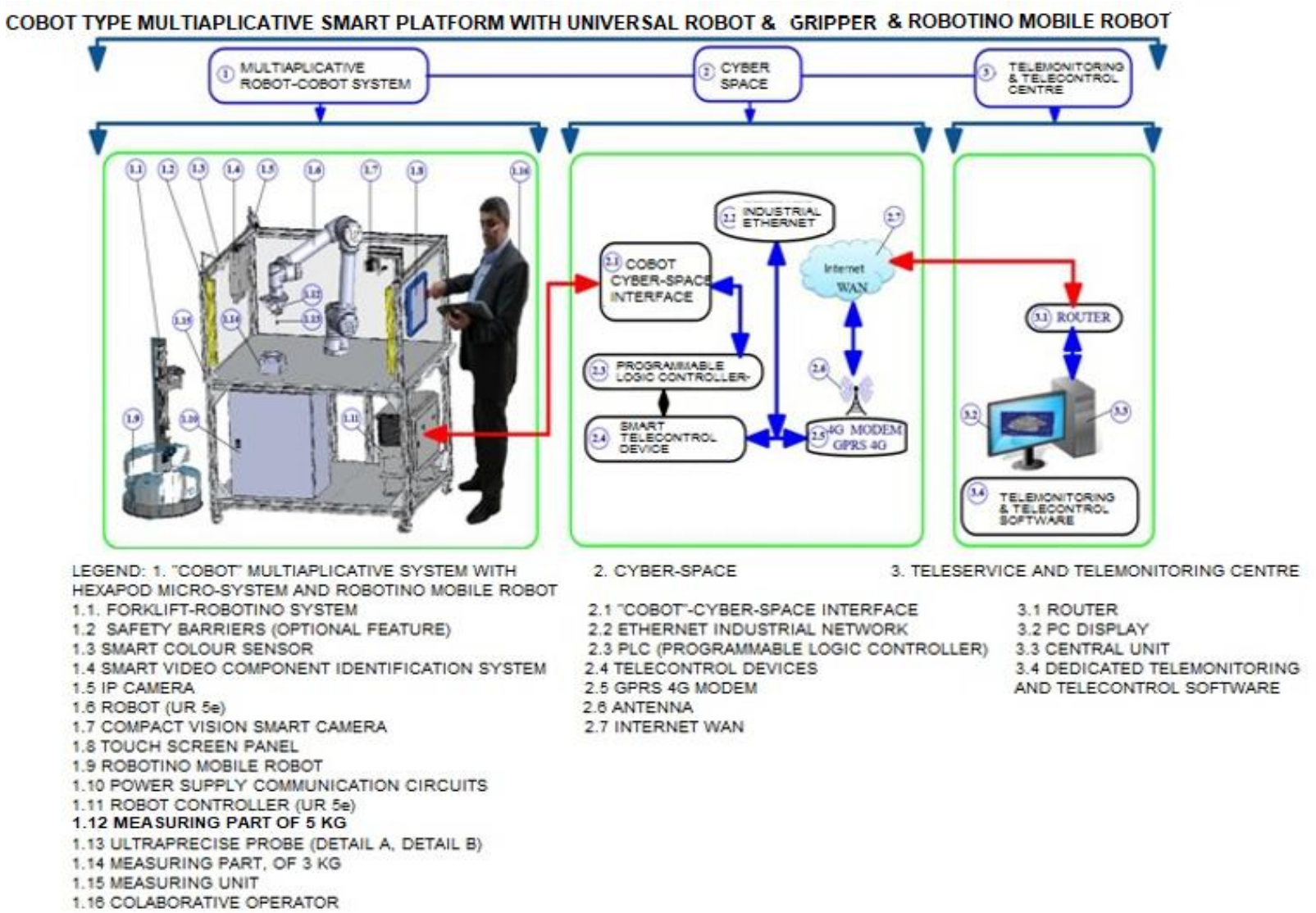

Figure 4

The system is additionally protected by infrared safety barriers (1.2) and communicates with the special equipment in the cabinet (1.11) both with the internal industrial bus (2.2) and with the WAN Internet network via the 4G GPRS modem. This communication connection connects to the remotecontrol centre (3) provided with a PC central unit (3.3) connected to the router (3.1) and on which the specialized software (3.4) runs.

The system can also be operated locally, using a pre-installed program by emulating working positions using the Touch-Screen Panel (1.8). The positioning of the final effector (3D scanner) can be done in a well-defined area of the entire workspace of the robot with 6 degrees of freedom (6DOF).

During the measurement process, the position information values - the measured values - are transmitted in numerical vector data packages for complex processing both in real time and subsequent processing too.

In addition to the innovative performance of robotic platforms, operator safety is another necessary prerequisite of successful operations under smart manufacturing process. To avoid risky situations, before the emergence of robots, industrial robots have been kept away from workers through a series of protection measures since the beginning of the history of automation. Collaborative robots have completely changed the paradigm and allowed the implementation of cobotic platforms, without a safety infrastructure that requires high costs and space. The main competitive advantages of UR cobots include easy operation, fast deployment and an interface that allows the collaborative operator (1.16) to take on a form of intuitive programming, which can also be performed by direct physical contact with the robotic arm or via the touch-screen. Cobots are able to work in close proximity to humans without safety barriers, but this approach requires full control over the speed, force, power, and momentum generated by the cobots during their operations. Even though UR cobots are at the top of safety standards, the multi-application cobot platform has also been provided with optical safety barriers, as an additional adaptive function for a wide range of applications.

The connection to the cyberspace (2) is made through a specialized interface (2.1) that allows communication with the robot controller (1.11) the data being adapted to be transferred to a Programmable Logic (PLC) (2.3) used to automate mechatronic processes and for storing the working variables of the specific control program, as well as to receive - in a synchronized way - information from the smart remote control equipment (2.4) implemented either with microcontroller via RISC architecture, or via FPGA, and which connect the PLC and the $4 \mathrm{G}$ telecommunication modem using a specific RS232 or USB protocol. 
Various public or private networks can be used to access the 4G connection. Various modems (2.3) can be used for connectivity, and they are all compatible with VPN protocols. Event-driven, or cyclic data processing is performed using special remotecontrol protocols and allows the operating personnel to efficiently manage the process as a whole, or in detail.

The Remote-Control Centre (3) provides the connection to the WAN Internet (2.7) using a router (3.1) equipped with VPN function to ensure a secure data encryption.

A second level of security can be studied by using its own encryption algorithm and by specifically configuring the FIREWALL function of the operating system on the PC (OS: Windows or Linux). The computer (3.3) in the Telemonitoring Centre (3) runs a software specially designed to perform the functions of remote control, telemonitoring and teleservice.

\section{Conclusions}

These smart systems and platforms of СОВOT type, are integrated in the processes of the Smart Industry (4.0), constituting the digital part of the Enterprise, of the Smart Industry and, respectively, of the post-computerized society.

The expansion and development of smart systems and platforms is the first step in the digitalization and intelligence of the machine building industry in Romania.

\section{References}

[1] Anghel Constantin, „Telementenance and Teleservice Oriented Design of Dependable Mechatronic System in Automotive Industry", Proceedings of the International Conference on Numerical Analysis and Applied Mathematics 2014 (ICNAAM-2014), AIP Conference Proceedings 1648, 620004 (2015); doi: http://dx.doi.org/10.1063/1.4912854

[2] Gheorghe, G., Bajenaru V., Ilie, I., Ingineria Mecatronică și Cyber-MixMecatronică pentru Construcția Intreprinderii Digitale și Industriei Inteligente (4.0), Bucharest, CEFIN Pubishing House, (2019)

[3] Gheorghe, G., Concept and Mechatronics and Cyber-Mixmechatronics Constructions, Integrated in COBOT Type Technology Platform for Intelligent Industry (4.0), Proceedings of the International Mechatronics and CyberMixMechatronics, Springer Link, Publishing House (2019),

[4] Costa, D., Martins M., Martins,S., Teixeira, E., Bastos, A. Cunha, A.R., Varela, L. and Machado, J., Evaluation of Different Mechanisms of Production Activity Control in the Context of Industry 4.0, Proceedings of the International Conference of Mechatronics and CyberMixMechatronics, Springer Link Publishing House (2019),

[5] Gheorghe, Challenges and Research in the Innovation of Digital Enterprise and Smart Industry (4.0), Proceedings of 2019 International Conference on Hydraulics and Pneumatics HERVEX, November 13-15, Baile Govora, Romania, ISSN 1454 - 8003 (2019). 